\title{
PROGRAMA PARA FORTALECER FACTORES PROTECTORES QUE LIMITAN EL CONSUMO DE TABACO Y ALCOHOL EN ESTUDIANTES DE EDUCACIÓN MEDIA ${ }^{1}$
}

\author{
Santiaga Enriqueta Esparza Almanza ${ }^{2}$ \\ Sandra Cristina Pillon ${ }^{3}$
}

Almanza SEE, Pillon SC. Programa para fortalecer factores protectores que limitan el consumo de tabaco y alcohol en estudiantes de educación media. Rev Latino-am Enfermagem 2004 março-abril; 12(número especial):324-32.

Los programas dirigidos a adolescentes indican que las intervenciones más efectivas para prevenir el consumo de tabaco y alcohol incluyen entrenamiento en habilidades sociales. En el presente estudio se desarrolló una intervención educativa encaminada a fortalecer los factores protectores autoestima y asertividad, aplicando canales de prevención primaria. El Modelo de Etapas Motivacionales de Componentes Múltiples y la Teoría del Aprendizaje Social brindan sustento teórico al presente estudio. Se realizaron dos mediciones, antes, y después de haber concluido la intervención. Resultados: se encontró un incremento de autoestima de $M e=63.14$ en la primer medición a Me=84.38 al finalizar la intervención. El nivel de asertividad se incrementó significativamente de $M e=60.09$ a 81.26 al finalizar la intervención en el grupo experimental ( $p<.001)$. Las medias de autoestima y asertividad fueron significativamente mayores en el grupo experimental que en el grupo control ( $p<.001)$.

DESCRIPTORES: autoestima; asertividad; estudiante; educación media; tabaco; alcohol; enfermería

\section{PROTECTIVE FACTOR PROMOTION PROGRAM WITH A VIEW TO LIMITING TOBACCO AND ALCOHOL CONSUMPTION AMONG SECUNDARY STUDENTS}

Adolescent-oriented programs indicate that the most effective interventions to prevent tobacco and alcohol consumption include social ability training. This article reports on an educational intervention aimed at strengthening protective factors, selfesteem and assertiveness, applying primary prevention channels, based on the Multi-Component Motivational Stages theoretical model and Social Learning Theory. Measurements were made before and after the intervention. Results: we verified increased self-esteem scores with $M e=63.1$ at first measurement and Me=84.38 at the end of the intervention. A significant increase in assertiveness occurred after the intervention, with Me rising from 60 to $81.2(p<.001)$. Average scores for self-esteem and assertiveness were significantly higher in the experimental group when compared to the control group ( $p<.001)$.

DESCRIPTORS: self-esteem; assertiveness; student; secondary education; tobacco; alcohol; nursing

\section{PROGRAMA PARA FORTALECER FATORES PROTETORES QUE LIMITAM O CONSUMO DE TABACO E ÁLCOOL ENTRE ESTUDANTES DE EDUCAÇÃO MÉDIA}

Os programas dirigidos aos adolescentes indicam que as intervenções mais efetivas para prevenir o consumo de tabaco e álcool incluem treinamento das habilidades sociais. No presente estudo desenvolveu-se uma intervenção educativa destinada a fortalecer os fatores protetores, autoestima e assertividade, aplicando canais de prevenção primária. O Modelo de Etapas Motivacionais de Componentes Múltiplos e a Teoria do Aprendizado Social proporcionam sustento teórico no presente estudo. Foram realizadas mensurações antes e depois de ter concluído a intervenção. Resultados: encontrou um aumento de autoestima com $\mathrm{Me}=63.1$ na primeira mensuração $\mathrm{Me}=84.38$ ao final da intervenção. No nível de assertividade se aperfeiçoou significativamente de $\mathrm{Me}=60$ a 81.2 ao finalizar a intervenção no grupo experimental ( $p<.001)$. As médias de autoestima e assertividade foram significativamente maiores no grupo experimental comparado com o grupo controle ( $p<.001)$.

DESCRITORES: autoestima; assertividade; estudante; educação média; tabaco; álcool; enfermagem

\footnotetext{
${ }^{1}$ Las opiniones expresadas en este articulo son de responsabilidad exclusiva de los autores y no representan la posición de la organización donde trabajan o de su administración; ${ }^{2}$ Investiagadora, Licenciado en Enfermería, Profesor de la Universidad Autónoma de Nueva León, correo electrónico: sesparza54@hotmail.cpm; ${ }^{3}$ Docente de la Escuela de Enfermería de Ribeirão Preto, de la Universidad de São Paulo, Centro Colaborador de la OMS para el desarrollo de la investigación en enfermería, correo electrónico: pillon@eerp.usp.br
} 
INTRODUCCIÓN

En México el uso de sustancias adictivas constituye un fenómeno complejo que tiene consecuencias adversas en la salud individual y familiar por lo que el profesional de enfermería tiene una responsabilidad en la prevención primaria del uso de drogas. Los resultados de la Tercer Encuesta Nacional de Adicciones (ENA) ${ }^{(1-2)}$, indican que, si bien la prevalencia del uso de drogas en México es aún baja en comparación con la de otros países de América Latina, se registran incrementos y tendencias que son preocupantes. Para 1998 se reportó que el 12.9\% de los adolescentes de 12 a 17 años son fumadores, y el $27.6 \%$ consume bebidas alcohólicas, se señala además que uno de cada tres menores de edad entre 12 y 17 años, refirieron haber consumido alguna vez en su vida una copa de alcohol, el 5\% indicó consumir cinco copas o más durante la semana previa a la encuesta, tres de cada 100 menores de edad informaron haber llegado a la embriaguez ${ }^{(2)}$.

Los programas de prevención dirigidos a adolescentes, indican que las intervenciones más efectivas para modificar el consumo de tabaco y alcohol, son los que incluyen actividades de entrenamiento en habilidades sociales para rechazar la presión del grupo de iguales y así rechazar la oferta de drogas ${ }^{(3-4)}$. En continuidad con estos hallazgos el Modelo de Etapas Motivacionales de Componentes Múltiples (McMOS) y la Teoría del Aprendizaje Social (TAS) brindan sustento teórico para el desarrollo de programas de prevención del tabaco y alcohol.

El McMOS ${ }^{(5)}$ plantea etapas de adquisición del habito de drogas (precontemplación, contemplación, preparación, acción y mantenimiento), las primeras tres etapas de adquisición representan oportunidades para la prevención primaria, donde la meta está dirigida a reducir la facilidad o motivación para iniciar una conducta que dañe la salud. En el fenómeno de las drogas la meta es retardar el inicio al consumo, es decir que el individuo no piense en usar la droga (precontemplación), ó revertir el movimiento de etapas desde un patrón de uso regular (mantenimiento) hasta no pensar en usar la sustancia.

EI McMOS plantea que los objetivos, estrategias y contenido de las intervenciones variarán en la prevención primaria de acuerdo a la etapa de adquisición de hábito en que se encuentre el estudiante y a los factores de riesgo presentes, lo anterior tiende a motivar con mayor éxito a los jóvenes para el cambio, al proporcionar intervenciones más individualizadas. Se ha encontrado que los constructos de la TAS están asociados a las etapas de adquisición del consumo de drogas ${ }^{(5-6)}$.

La TAS indica que una manera de ampliar la capacidad conductual en la persona para evitar el consumo de drogas es proporcionar conocimiento, práctica y retroalimentación relacionada con la realización de conductas para resistir las presiones sociales a usar drogas y habilidades de vida como asertividad ${ }^{5}$ Así mismo, se ha argumentado que la autoestima y la asertividad, pueden contribuir a mejorar la resistencia de los adolescentes a la presión del grupo de iguales para consumir drogas ${ }^{(7-8)}$.

La asertividad ha sido considerada como el índice más característico de las habilidades sociales y como la más importante entre todas ellas, la asertividad es definida como la capacidad para expresar lo que se cree, piensa y siente de manera directa y clara en un momento oportuno. Es la conducta interpersonal que implica la expresión directa de los propios sentimientos y la defensa de los propios puntos de vista sin negar la de otros. Así mismo, se puede decir que la asertividad es la capacidad de poner límites y de mantenerlos, es un estilo de comportamiento que permite actuar pensando en el bienestar propio, ejerciendo los derechos de uno y respetando a la vez los derechos de los demás ${ }^{(8-9)}$.

Algunos estudios transversales muestran la asociación entre asertividad y consumo de tabaco y alcohol $^{(4,8)}$. El deseo de ser aceptado y pertenecer a un grupo es fundamental durante la adolescencia, y para manejar las presiones que implícitamente tienen los grupos hacia los miembros, es necesario desarrollar ciertas habilidades que permita a los adolescentes identificar cuando están siendo presionados para realizar una acción con la cual no se sienten cómodos y saber elegir independientemente de lo que el grupo de amigos desee ${ }^{(9)}$.

Otro de los constructos de la TAS es el de respuestas de enfrentamiento emocional definido como estrategias para tratar los estímulos emocionales ${ }^{(5)}$. En este estudio se aborda la autoestima como elemento que fortalece la adquisición de habilidades del estudiante para prevenir el uso de drogas. El tener un buen nivel de autoestima es uno de los recursos mas valiosos en los adolescentes, un adolescente con autoestima alta aprende más eficazmente, desarrolla relaciones mucho mas gratas, esta mas capacitado para aprovechar las oportunidades que se le presenten, para trabajar productivamente y ser 
autosuficiente, posee una mayor conciencia del rumbo que sigue. $Y$ lo que es mas, si el adolescente termina esta etapa de su vida con un autoestima fuerte y bien desarrollada podrá entrar en la vida adulta con buena parte de los cimientos necesarios para llevar una existencia productiva y satisfactoria $^{(7)}$.

Por otra parte los programas de prevención de alcohol y drogas en general emplean estrategias que asumen son generalizables a una sociedad entera, sin embargo se ha encontrado que estas estrategias no se enfocan a las diferencias individuales entre los jóvenes respecto a los niveles de riesgo al uso de drogas, de igual forma no se toma en cuenta la heterogeneidad de la población objetivo. Por lo que es imperante la necesidad de ampliar las intervenciones preventivas individualizándolas a etapas particulares de adquisición y factores de riesgo/protección presentes.

La implicación para los educadores de salud es que deben desarrollar intervenciones que apunten a los factores protectores que influyen en la categoría de etapa de adquisición de los jóvenes ${ }^{(5)}$. El modelo McMOS propone un marco para identificar las diferentes formas de acercamiento al individuo, es decir relacionar las etapas de adquisición de drogas con las formas de prevención y mensajes a lo cual llama canales de prevención, cada uno de estos canales de prevención tienen fortalezas únicas que pueden motivar a los jóvenes a evitar el uso de drogas.

Los canales que influyen en la etapa de precontemplación son los medios y materiales impresos, los canales interpersonales tales como los contactos personales en la cual se incluye el programa educativo y el consejo individual se piensa aumentan significativamente la recepción e integración de los mensajes y comúnmente se utilizan para enseñar y modelar las habilidades para el cambio conductual relacionado a la prevención del uso de drogas $^{(5-10)}$.

En este contexto se ha documentado que las intervenciones preventivas individualizadas a factores de protección específicos que involucran manejo de materiales junto con intervención educativa de enfermería pueden producir resultados significativamente más positivos que el solo brindar información ${ }^{(11)}$.

Finalmente, intervenciones desarrolladas en estudiantes en donde se involucra la atención directa del profesional de la salud, con población específica en alguna etapa de adquisición y con abordaje de factores de protección, favorecen la resistencia del adolescente al consumo de drogas, y existe evidencia de reducciones de corto plazo en el consumo de alcohol cuando se imparten sesiones educativas comparado con intervenciones en donde se brinda solo material impreso ${ }^{(10-}$ ${ }^{12)}$. Estudios realizados en Monterrey indican que una gran parte de los jóvenes se encuentran en la etapa de adquisición precontemplación al consumo de tabaco y alcohol, por lo que la prevención primaria específica a la etapa de precontemplación es importante para evitar el inicio al consumo de sustancias. En este mismo contexto la prevención primaria es una oportunidad para que la enfermera escolar desarrolle acciones encaminadas a evitar el uso y abuso de tabaco y alcohol, y que tome una posición en el sistema educativo y de salud en el estado de Nuevo León.

Con base en los datos antes señalados, el propósito del presente estudio fue medir el efecto de una intervención educativa para fortalecer factores protectores (autoestima, asertividad) para prevenir el consumo de tabaco y alcohol en los estudiantes de educación media básica.

En el presente estudio se desarrolló una intervención educativa encaminada a fortalecer los factores protectores de autoestima y asertividad, para la prevención del uso de tabaco y alcohol, aplicando canales de prevención primaria (uso de materiales impresos, y el canal interpersonal). Se plantearon dos hipótesis de investigación para el presente estudio.

\section{HIPÓTESIS}

H1. La autoestima presentará un incremento mayor en el grupo de intervención que en el grupo de comparación.

H2. El grupo de intervención presentará puntajes de asertividad más altos que el grupo de comparación.

\section{METODOLOGÍA}

Diseño del estudio: Casí-experimental, de mediciones repetidas ${ }^{(13)}$, ya que se desarrolló una intervención educativa, en el grupo experimental y se desarrolló una acción puntual en el grupo de comparación. Se realizaron dos mediciones, antes y después de haber concluido la intervención. 
Grupo Experimental:

$\mathrm{O}_{1} \mathrm{X}_{1} \mathrm{X}_{2} \mathrm{X}_{3} \mathrm{X}_{4} \mathrm{X}_{5} \mathrm{X}_{6} \mathrm{O}_{2}$

Intervención educativa, canal interpersonal y material impreso.

Grupo Comparación:

$\mathrm{O}_{1}$ Entrega de material empreso $\mathrm{O}_{2}$

$\mathrm{O}=$ Medición $\quad \mathrm{X}=$ Sesión de intervención

La población del grupo experimental y de comparación fueron estudiantes de ambos sexos que cursan el primer grado de educación media básica. Para evitar el sesgo de contaminación, los participantes del grupo control fueron de una institución educativa diferente a la del grupo experimental. Dado que las estrategias de abordaje varían en cada etapa de adquisición, para el presente estudio solo se consideró a los estudiantes de secundaria que se encontraron en la etapa de adquisición precontemplación.

Local: Las sesiones educativas se llevaron a cabo en las instalaciones de la Institución Educativa localizada en una colonia urbana del Municipio de Apodaca, Nuevo León, México, esta localidad es de bajo nivel socioeconómico. Muestreo y muestra: El tamaño de la muestra estuvo conformada por 40 estudiantes para el grupo experimental y 40 estudiantes para el grupo control. En el tamaño de la muestra se cuidó el $10 \%$ de deserción. El muestreo fue aleatorio estratificado por cada grupo de primer año (3). Se seleccionaron a 60 estudiantes de primer grado, de éstos se eligieron a 40 estudiantes que cumplían los criterios de inclusión, para el grupo experimental y 40 estudiantes del grupo comparación, en cada escuela secundaria.

Criterio de exclusión: Estudiantes que se encontraron en etapa contemplación, preparación, acción y mantenimiento al consumo de tabaco y/o alcohol. Estudiantes que se encontraron en etapa de contempación, preparación, acción o mantenimiento al consumo de drogas ilícitas. Estos datos se identificaron a través de un cuestionario filtro.

Mediciones: En primer lugar se aplicó la cedula de datos personales que indaga edad, sexo. Como instrumento Filtro se utilizó el instrumento estructurado denominado Encuesta de alcohol y Salud de los Jóvenes ${ }^{(14)}$ con el fin de identificar el consumo de tabaco, alcohol y drogas ilícitas (marihuana, cocaína) alguna vez en la vida, fue necesario agregar los items para el consumo de inhalables, por ser una de las drogas que puede ser consumida por la población a estudiar. Estos datos fueron utilizados de manera descriptiva solamente y para la selección de estudiantes. Además se indagó la cantidad y frecuencia del consumo de tabaco, alcohol y drogas ilícitas en los últimos 15 días, y a través de cinco reactivos se identificó la etapa de adquisición al consumo en que se encuentra.

Se aplicó la Escala de Autoestima ${ }^{(15)}$ que mide la autoestima como aquellos sentimientos de respeto y aceptación hacia uno mismo. Dicha escala está constituida por 10 reactivos, con escala de respuesta de cuatro opciones, ordenadas desde una connotación mas positiva (estoy muy de acuerdo), hasta la mas negativa (Estoy muy en desacuerdo). Se aplico también la Escala de Asertividad ${ }^{(16)}$ esta escala tiene como objetivo evaluar el comportamiento social autoafirmativo de la persona. $\mathrm{Ha}$ sido utilizada en estudiantes de nivel medio y medio superior con un alpha de Cronbach de $.81^{(17)}$. Actualmente se utiliza como un instrumento confiable para medir el estado de asertividad. Compuesta por 30 items, fue necesario adaptar algunos reactivos de la escala con términos entendibles al lenguaje y actividades del estudiante de nuestra región. La escala original tiene respuesta dicotómica de falso y verdadero, sin embargo para evitar el sesgo de complacencia, se utilizó una escala de respuesta de cuatro opciones donde 1 indica nunca, 2 algunas veces, 3 frecuentemente y 4 siempre.

Medidas de control: Los participantes del grupo control y del grupo experimental pertenecen a escuelas diferentes, son de un mismo grado de secundaria y el mismo nivel socioeconómico. Un facilitador brindó la intervención y un profesional fungió como observador, cuidando el tiempo destinado para cada actividad de la sesión. El facilitador tiene formación de maestría en enfermería, participa en el proyecto prevención de adicciones y tiene experiencia con trabajo preventivo en adolescentes. Se brindó capacitación al faciliatdor para la ejecución de la intervención.

El material desarrollado para la intervención incluyó la bitácora de procedimientos que guía al facilitador en el desarrollo de las sesiones educativas, este manual incluye el objetivo de cada una de las sesiones, contenido, actividades de aprendizaje. Se desarrolló también el manual para el participante que incluye información importante de los temas que se abordaron en cada una de las sesiones, así como la guía de ejercicios que se desarrollaron en la sesiones, este manual se entregó a cada uno de los participantes del grupo experimental.

Se tuvo especial cuidado en que los contenidos temáticos abordados en la intervención, estén acordes a la definición de los conceptos teóricos. Algunos de los ejercicios propuestos se tomaron del Manual "Habilidades para la vida"(13). 


\section{RESUMEN DE INTERVENCIÓN}

La intervención tuvo una duración de seis semanas, el grupo experimental recibió seis sesiones de frecuencia semanal con una duración de una hora. Las sesiones se llevaron acabo en una de las aulas de la institución educativa, cuidando que solo los participantes estuvieran presentes en la misma. Durante la primer sesión se entregó un manual educativo impreso, cuyo contenido esta encaminado a incrementar el autoestima, la asertividad, y los conocimientos acerca de las consecuencias del consumo de tabaco y alcohol, contiene además actividades para desarrollar durante las sesiones educativas. En las primeras dos sesiones se abordaron los temas Autoestima, tipos de calidades, características de la persona con autoestima positiva y negativa, influencia del autoestima en el adolescente, ingredientes y actitudes que sostienen mi autoestima. En la tercer y cuarta sesión de intervención se abordan temas de asertividad, tipos de respuestas ante la presión, beneficios de ser asertivo, como resistir la presión de grupos, como tomar una decisión positiva para tu vida, por ultimo en la quinta y sexta sesión se tratan las consecuencias del consumo de drogas en general y específicamente consecuencias del tabaco y alcohol, mitos y realidades del consumo de tabaco y alcohol, vivir una vida sin alcohol y tabaco.

Durante las sesiones educativas se aplicaron estrategias de enseñanza que favorecen el objetivo de las sesiones de una manera dinámica, motivacional, e interactiva, y que favorezca la desinhibición grupal. Algunas de estas son: actividad "El árbol de mi autoestima". Reflexión "Mostrando mis fortalezas", Dinámica "Mi compromiso con la salud", Collage "Consecuencias del alcohol", Dramatización "Como decir Si, Cómo decir No", Ejercicio "Mitos y realidades"(18). Las sesiones se realizaron en un aula del plantel educativo, para lo cual se adaptó el ambiente del lugar, de manera que el contexto (ruido, falta de privacidad) no interfiriera con la reacción del estudiante ante la intervención y facilite el clima de confianza entre los participantes y el facilitador.

Al finalizar la intervención se realizó una segunda medición de las variables de estudio. En el grupo control se realizó la primer medición en una semana aplicando los tres instrumentos ya mencionados, se hizo entrega a cada estudiante de un tríptico impreso con información acerca de las consecuencias del consumo de tabaco y alcohol. Durante la misma semana de la segunda medición del grupo experimental se levantó la segunda medición del grupo control.

Para el proceso estadístico de los datos se utilizó el paquete estadístico SPSS/Win versión 10 (Statistical Package for the Social Sciences for Windows), se obtuvieron estadísticas descriptivas frecuencias, porcentajes, medidas de tendencia central y dispersión, de las escalas de autoestima y asertividad se obtuvieron; para el sustento de las hipótesis se aplicó el estadístico diferencia de medias.

El presente estudio se apegó a los principios éticos relacionados con el proceso al que estuvieron expuestos los participantes del estudio, conforme a lo establecido en el Reglamento de la Ley General de Salud en Materia de Investigación para la Salud ${ }^{(18)}$.

\section{RESULTADOS}

Los resultados del estudio de intervención se presentan en el siguiente orden: en primer lugar los datos descriptivos de los participantes del grupo experimental y el grupo de comparación, en segundo lugar los datos descriptivos de las variables del estudio, y por ultimo la estadística inferencial para prueba de hipótesis.

Características de los participantes del estudio

Tabla 1 - Presentación de frecuencia y proporciones de edad de los participantes

\begin{tabular}{|c|c|c|c|c|}
\hline \multirow[t]{2}{*}{ Edad } & \multicolumn{2}{|c|}{ Grupo Control $n_{1}$} & \multicolumn{2}{|c|}{ Grupo Experimental $n_{2}$} \\
\hline & $N$ & $\%$ & $\mathrm{~N}$ & $\%$ \\
\hline 12 años & 12 & 34.3 & 13 & 37.1 \\
\hline 13 años & 23 & 65.7 & 22 & 62.9 \\
\hline Total & 35 & 100 & 35 & 100 \\
\hline
\end{tabular}

La edad de los participantes del estudio osciló entre los 12 y 13 años. La edad media de los participantes fue de 12.6 años (DE=.48).

Tabla 2 - Distribución de la muestra frecuencia y proporción de sexo de los participantes

\begin{tabular}{ccccc}
\hline Sexo & \multicolumn{2}{c}{ Grupo Control $\mathrm{n}_{1}$} & \multicolumn{2}{c}{ Grupo Experimental $\mathrm{n}_{2}$} \\
& $\mathrm{~N}$ & $\%$ & $\mathrm{~N}$ & $\%$ \\
\hline Masculino & 23 & 65.7 & 20 & 57.1 \\
Femenino & 12 & 34.3 & 15 & 42.9 \\
\hline Total & 35 & 100 & 35 & 100 \\
\hline
\end{tabular}

Tanto en el grupo control como en el experimental prevaleció el sexo masculino con un $65.7 \%$ y $57.1 \%$. 
Datos descriptivos de las variables de estudio

Tabla 3 - Datos descriptivos de las variables de estudio grupo control y grupo experimental

\begin{tabular}{lcccccc}
\hline \multicolumn{1}{c}{$\begin{array}{l}\text { Valor } \\
\text { Mínimo }\end{array}$} & $\begin{array}{c}\text { Valor } \\
\text { Máximo }\end{array}$ & Media & $\begin{array}{l}\text { Desviación } \\
\text { Estándar }\end{array}$ & K-S Z & $\begin{array}{l}\text { Valor } \\
\text { de p }\end{array}$ \\
\hline $\begin{array}{l}\text { Grupo Control Primera } \\
\text { Autoestima }\end{array} \quad 30.00$ & 93.33 & 61.80 & 16.59 & .618 & .839 \\
Asertividad & 38.89 & 82.22 & 56.28 & 9.18 & .948 & .330 \\
Grupo Control Segunda Medición & & & & \\
Autoestima & 33.33 & 93.33 & 64.85 & 16.71 & .778 & .580 \\
Asertividad & 37.78 & 84.44 & 55.46 & 8.90 & .666 & .767 \\
Grupo Experimental Primera Medición & & & \\
Autoestima & 26.67 & 86.67 & 63.14 & 13.30 & .794 & .533 \\
Asertividad & 48.89 & 75.56 & 60.09 & 6.81 & .456 & .986 \\
Grupo Experimental Segunda Medición & & & \\
Autoestima & 76.67 & 100.0 & 84.38 & 6.14 & 1.12 & .157 \\
Asertividad & 58.89 & 94.44 & 81.26 & 7.08 & .590 & .877 \\
\hline
\end{tabular}

Los participantes del grupo control y del grupo experimental presentaron medias de autoestima mas bajas ( $M=61.80,63.33)$ durante la primer medición. De forma semejante se comportó el nivel de asertividad, ya que el grupo control presentó una media de $\mathrm{M}=55.55(\mathrm{DE}=9.18)$ y el grupo experimental una $\mathrm{M}=60.00(\mathrm{DE}=6.81) \mathrm{de}$ acuerdo con la Tabla 3. Los resultados de la prueba Kolmogorov-Smirnov muestran que los datos de las variables presentaron una distribución normal, lo que permitió aplicar la estadística paramétrica.

Tabla 4 - Presentación de las escalas de asertividad y autoestima aplicadas para grupo experimental y grupo control

\begin{tabular}{llcc}
\hline & Medición & $\begin{array}{c}\text { Grupo } \\
\text { Experimental } \\
(\mathrm{N}=35)\end{array}$ & $\begin{array}{c}\text { Grupo } \\
\text { Controle } \\
(\mathrm{N}=35)\end{array}$ \\
\hline Escala de Assertividade & $1^{\circ}$ y $2^{\circ}$ & $\mathrm{t}=14,55 \mathrm{p} .001$ & $\mathrm{t}=.411 \mathrm{p} .684$ \\
Escala de Autoestima & $1^{\circ}$ y $2^{\circ}$ & $\mathrm{t}=-8,86 \mathrm{p} .001$ & $\mathrm{t}=8,14 \mathrm{p} .421$ \\
\hline
\end{tabular}

La Tabla 4 presenta las diferencias entre una aplicación de escala de asertividad y de autoestima entre la primera y segunda medición para el grupo experimental y el grupo control. Las cuales representan valores significativos $(\mathrm{p} .=000)$ para ambas escalas del grupo experimental.

Tabla 5 - Diferencia de medias para autoestima en grupo experimental y grupo control

\begin{tabular}{|c|c|c|}
\hline Autoestima & $\begin{array}{c}\text { Grupo experimental } \\
(\mathrm{N}=35)\end{array}$ & $\begin{array}{l}\text { Grupo Control } \\
(N=35)\end{array}$ \\
\hline $\begin{array}{l}\text { Autoestima } \\
\text { Segunda Medición }\end{array}$ & $\begin{array}{cl}\text { Media } & \begin{array}{l}\text { Desviación } \\
\text { Estándar }\end{array} \\
\end{array}$ & $\begin{array}{ll}\text { Media } & \begin{array}{l}\text { Desviación } \\
\text { Estándar }\end{array} \\
\end{array}$ \\
\hline & $\begin{array}{ll}84.38 & 6.14\end{array}$ & $\begin{array}{ll}64.85 & 16.71\end{array}$ \\
\hline
\end{tabular}

Diferencia de Medias $=6.46, p=.001$
Los resultados de la Tabla 5 indican que la media de autoestima fue mayor en el grupo experimental con diferencias significativas $(t=6.46, p<.001)$ por lo que se sustenta la hipótesis de que la autoestima es mayor en el grupo de intervención que en el grupo de comparación.

Tabla 6 - Diferencia de medias asertividad grupo experimental y grupo control

\begin{tabular}{|c|c|c|c|c|}
\hline \multirow[b]{2}{*}{$\begin{array}{l}\text { Asertividad Segunda } \\
\text { Medición }\end{array}$} & \multicolumn{2}{|c|}{$\begin{array}{c}\text { Grupo Experimental } \\
(\mathrm{N}=35)\end{array}$} & \multicolumn{2}{|c|}{$\begin{array}{l}\text { Grupo Control } \\
(\mathrm{N}=35)\end{array}$} \\
\hline & Media & $\begin{array}{l}\text { Desviación } \\
\text { Estándar }\end{array}$ & Media & $\begin{array}{l}\text { Desviación } \\
\text { Estándar }\end{array}$ \\
\hline & 81.26 & 7.08 & 55.46 & 8.90 \\
\hline
\end{tabular}

Diferencia de Medias $=13.71, p=.001$

En la Tabla 6, se puede observar que la media de asertividad del grupo experimental es significativamente mayor que la media obtenida en la segunda medición del grupo control $(t=13.71, p<.001)$.

\section{DISCUSIÓN}

En el presente estudio fue posible diseñar, ejecutar y evaluar una intervención educativa que integra el componente de autoestima y asertividad como factores protectores para prevenir el consumo de tabaco y alcohol en estudiantes de secundaria, que se encuentran en etapa de precontemplación.

Los resultados obtenidos fueron satisfactorios al final de la intervención educativa comparados con las mediciones preliminares realizadas antes de la intervención. El grado de autoestima que presentaron los estudiantes de secundaria en la primer medición fue bajo ( $M e=63.14, D E=13.30)$; estos resultados fueron muy semejantes a lo reportado en una muestra de adolescentes $(\mathrm{Me}=63.14)$ con características semejantes a los participantes de este estudio. Sin embargo fueron bajos comparados con lo encontrado por Navarro y Portillo ${ }^{(19)}$ quien reportó que un $77 \%$ de adolescentes colombianos tienen un buen nivel de autoestima, y solo el $7.1 \%$ presentó un nivel de autoestima bajo. Por su parte Ruiz ${ }^{(20)}$ encontró en adolescentes una media de autoestima de 79.47, estas diferencias pueden deberse a que estos estudios fueron realizados en adolescentes de otros países y que probablemente se incluyen contenidos académicos que favorecen la autoestima, en etapas mas tempranas de formación. El nivel de estima encontrado en los participantes de este estudio afirman la ausencia de visión 
saludable que tienen de si mismos, lo cual es preocupante ya que son sujetos que están en un período crucial de su desarrollo, en donde una baja autoestima los convierte en población más susceptible para el inicio de una conducta adictiva.

El índice de asertividad arrojado en la primer medición del grupo experimental también fue bajo $(\mathrm{Me}=$ $60.09, \mathrm{DE}=6.81$ ), estos datos son similares con Garaigordobil $^{(21)}$ quien encontró en adolescentes de $12 \mathrm{a}$ 14 años que participaron en un programa educativo una media de asertividad de 59.52. En cambio Suelves y Sánchez ${ }^{(8)}$ al estudiar la relación de asertividad y uso de sustancias en adolescentes encontraron un nivel de asertividad alto. Aun y cuando los sujetos del estudio no consumen tabaco o alcohol, se puede conjeturar la influencia condicional que tiene el bajo nivel de asertividad ya que puede hacer que el sujeto sea mas vulnerable a otros factores, es decir se vuelve mas susceptible a la influencia de los amigos, lo cual en esta población especifica es un factor de riesgo para el consumo de drogas. Esto puede ser interpretado a la luz de los planteamientos del aprendizaje social, ya que un individuo con dificultades para expresar abiertamente sus opiniones o para desenvolverse adecuadamente en su medio social podrá ser mas vulnerable a las presiones de su medio.

Los resultados obtenidos en la medición final de las variables de estudio reflejan el efecto significativo que tuvo el programa educativo en los participantes del grupo experimental. Los sujetos del grupo experimental incrementaron su autoestima de $\mathrm{Me}=63.14$ a una media de 84.38 ( $D E=6.14)$. La habilidad de asertividad se incrementó de $\mathrm{Me}=60.09$ ( $\mathrm{DE}=6.81$ ), a $\mathrm{Me}=81.26, \mathrm{DE}=$ 7.08. Estos datos reflejan la capacidad del adolescente para la adquisición de nueva información y la estimulación del pensamiento creativo. En su estudio ${ }^{(20)}$, no encontró diferencia de la autoestima y asertividad entre la primer y segunda medición del grupo experimental, en cambio Garaigordobil $^{(21)}$ en el programa de asertividad y estrategias cognitivas de afrontamiento en situaciones sociales si muestra en sus hallazgos diferencias significativas en la medición preprueba y posprueba de asertividad.

Por su parte los resultados del grupo control no presentaron diferencias entre la primer y segunda medición de autoestima y asertividad. Los hallazgos positivos en el grupo experimental pueden deberse a varios elementos que se tomaron en cuenta para el diseño metodológico y la ejecución de la intervención. En primer lugar se puede decir que cuando un programa educativo se sustenta en un marco teórico en este caso el modelo de etapas de componentes múltiples, modelo especifico para la prevención primaria en el fenómeno de las drogas, es más efectivo que cuando se carece de él y se desarrolla un educación de manera tradicional. En una metanálisis ${ }^{(22)}$ que analiza el efecto de varias estudios de intervención en adolescentes, concluyen que los programas educativos bajo un enfoque teórico presentan mayor probabilidad de éxito.

En segundo lugar, como lo indica el McMOS, las estrategias de prevención y los mensajes transmitidos a los jóvenes es factible hacerlo a través de distintos canales. En el grupo experimental se emplearon los medios de comunicación, materiales y el canal interpersonal, a diferencia del grupo control donde solo se empleo el material impreso. En este contexto Werch ${ }^{(12)}$ reportó menor consumo de alcohol en jóvenes que tuvieron interacción y asesoría con profesionales de enfermería y medicina, a diferencia de aquellos que solo tuvieron a su alcance materiales informativos impresos.

Se deduce de esto la importante tarea que tiene el profesional de enfermería en el contexto comunitario y el ambiente escolar, al diseñar, operar y evaluar una intervención educativa con el fin de prevenir el consumo de drogas.

En tercer lugar las estrategias aplicadas en el programa educativo fueron acordes a lo recomendado por Werch y DiClemente ${ }^{(5)}$ para los conceptos de capacidad conductual y enfrentamiento emocional de la Teoría del Aprendizaje Social. La participación de los adolescentes del grupo experimental fue activa, esto pudo haber sido motivado por las distintas actividades aplicadas para obtener un aprendizaje significativo, que obliga a que el receptor no fuera un sujeto pasivo, pues implicó la interacción no solo con el profesional de enfermería, sino además con sus compañeros; las actividades encaminadas a disminuir actitudes pasivas y agresivas $y$ a incrementar las respuestas asertivas habilitaron a el estudiante en la asertividad.

Finalmente es importante reconocer que el contenido abordado en la intervención educativa habilitó al estudiante del grupo experimental no solo en la prevención del consumo de tabaco y alcohol, ya que el incremento de autoestima y asertividad favorecen la prevención de otras conductas de riesgo en el adolescente. 


\section{CONCLUSIONES}

Se puede concluir que los estudiantes de educación media del grupo control no presentaron diferencias de autoestima y asertividad entre la primer y segunda medición.

Los estudiantes de educación media del grupo experimental presentaron un nivel de autoestima mayor que en el grupo de comparación al término de la intervención.

El nivel de asertividad fue mayor en los estudiantes que participaron en el grupo experimental que en el grupo de comparación.

Los canales de prevención, medios y materiales impresos y el canal interpersonal fueron medios efectivos para incrementar la autoestima y la asertividad.

Los conceptos del aprendizaje social, capacidad conductual y enfrentamiento emocional fueron un marco efectivo para el sustento de la intervención educativa. Los resultados del estudio contribuyen al cuidado de la salud del grupo de adolescentes a través de la prevención del consumo de tabaco y alcohol.

Se recomienda realizar otras dos mediciones de autoestima y asertividad con los mismos intervalos de

\section{REFERENCIAS BIBLIOGRÁFICAS}

1. Consejo Nacional contra las Adicciones-Secretaría de Salud. El Consumo de Drogas en México: Diagnóstico, Tendencias y Acciones. México (DF). [Serie en red]. Disponible en: URL: http://www.ssa.gog.mx/unidades/concadic/CDM.htm 2. Tapia CR, Medina MM, Cravioto QP. Las adicciones: Dimensión, Impacto y Perspectivas. 2da. ed. México (DF): El Manual Moderno; 1998.

3. Secretaría de Salud (MX). Norma Oficial Mexicana NOM028-SSA2-1999, para la Prevención, Tratamiento y Control de las Adicciones. México: Secretaría de Salud; 1999.

4. Botvin G. Life Skills Training Program. Princeton (NJ): Princeton Health Press; 2001.

5. Werch E, DiClemente C, Modelo de Etapas con Componentes Múltiples para relacionar las estrategias y mensajes de prevención de drogas con la etapa de uso de los jóvenes. Health Educ Res, Theory and Practice 1994; $9(1): 1-21$.

6. Shrier LA, Haris SK, Sternberg M, Beardslee WR. Associations of depression, self-steem, and substance use with sexual risk among adolescents. Prev Med 2001 Sep; 33(3):179-89.

7. Qué relación existe entre el autoestima y el uso de drogas? [Serie en Red]. Disponible en: URL: www.Adcd.org/sp/drogas 8. Suelves J, Sánchez T. Asertividad y uso de sustancias en la adolescencia: Resultados de un estudio transversal. Anales de Psicología 2001; 17(1):15-22 medición, a la sexta semana y a los tres meses; tanto en el grupo experimental y de comparación para ver el efecto de la intervención a través del tiempo.

Aplicar los canales de prevención ejecutados en este estudio, medios interpersonales y medios impresos, con el fin de incrementar otros factores protectores que favorezcan la prevención del consumo de drogas en adolescentes.

Adaptar la intervención educativa a población adolescente en etapas de adquisición contemplación y preparación al consumo de drogas.

\section{AGRADECIMIENTOS}

Agradecemos a todos los docentes de la Escuela de Enfermería de Ribeirão Preto y a los treinta y tres participantes de nueve países latinoamericanos que participaron en el proceso de implementación de los programas, y también al Gobierno del Japón y al Programa de Becas de la OEA por su apoyo financiero y becas que posibilitaron la implementación del "I Programa Regional de Capacitación en Investigación para Enfermeros de América Latina".

9. Marsellach U. Técnicas de Entrenamiento en Asertividad: Debate; 1998.

10. Werch CH, Anzalone D, Brokiewicz L, Felker J, Carlson J, Castellon V. An Intervention for preventing alcohol use among inner city middle school students. Arch Family Med 1996; 5:14652.

11. Werch EC, Intervenciones Preventivas de Alcohol basadas en un modelo de etapas de Adquisición. Am J Health Beh 2001; 25(3):206-16.

12. Werch CE, Carlson JM, Pappas DM, DiClemente C. Brief nurse consultations for preventing alcohol use among urban school youth. J School Health 1996; 66(9):335-8.

13. Madrigal S, Sayago W. Manual de capacitación para docentes, habilidades para la vida: prevención del tabaco, alcohol y otras drogas. Caracas (Venezuela): Exlibris; 1999. 14. Werch $\mathrm{CH}$. Encuesta de Alcohol y Salud de los Jóvenes. Florida: University of north Florida, Center for Drug Prevention \& Health Promotion; 2001.

15. Rosenberg. Escala de Autoestima, 1965.

16. Rathus SA. A 30-item schedule for assessing assertive behavior. Behavior Ther 1973; 4:398-406.

17. Pasqualli V. Escala de Asertividad Rathus-Ras: Adaptación Brasileira. Psic.: Teoria y Pesqui 1990; 6(3).

18. Secretaria de Salud (MX). Ley General de Salud en Materia de Investigación. Leyes y Códigos de México. México: SS; 2000. 
19. Navarro HM, Pontillo CHV. Autoestima del adolescente y riesgo de consumo de alcohol. Actuali Enfermería 2002; 5(1):7-12.

20. Ruiz L. Un programa de prevención primaria y secundaria en los trastornos de conducta alimentaria en adolescentes. Intervención comunitaria en psicología. [on line]. Primer Congreso Virtual de psiquiatría. [Serie en Rede]. [citado en mayo de 2002]: Disponible en: URL: <http: www. psiquiatria. com/congreso>

21. Garaigordobil LM. Intervención con adolescentes: Impacto de un programa en la asertividad y en las estrategias cognitivas de afrontamiento de situaciones sociales. Psicol Conductual 2001; 9(2):221-46. 